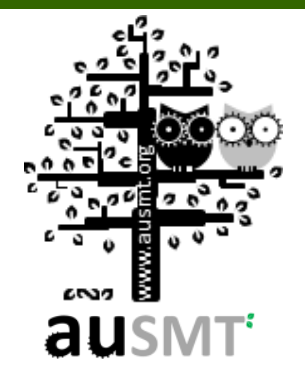

\title{
Using Focused Electron Beams to Drill Straight Nanopores on a Membrane
}

\author{
Yung-Cheng Wang1, *, Dau-Chung Wang1, and Tsan-Chu Lin' \\ ${ }^{1}$ Institute of Mechanical Engineering, National Yunlin University of Science and Technology, Taiwan \\ 2. Graduate School of Engineering Science and Technology, National Yunlin University of Science and Technology, Taiwan \\ (Received 13 January 2014; Accepted 23 May 2014; Published on line September 2014) \\ *Email: wangyc@yuntech.edu.tw \\ DOI: 10.5875/ausmt.v4i3.556
}

\begin{abstract}
A high-resolution focused electron beam is used for the fabrication of metal nanostructures and devices with insulating membranes by nanosculpting metal films. This top-down focused electron beam drilling method uses the controlled ablation of materials to produce nanoscale devices with near-atomic precision of order. Using the proposed procedure, nano-drilling is not directly realized through the aperture, but by using a focused electron beam to burn away the solvent. Recent studies have investigated silicon nitride nanopores with an hourglass profile and silica nanopores with a pyramid-shaped cross-section, but electronic drilling in these approaches failed to produce straight nanopores. A method is proposed to improve the membranes' thermal conductivity to rapidly produce straight nanopores, and is experimentally confirmed and has significant potential for use in nano-sensors or nano-devices.
\end{abstract}

Keywords: Nanopores; electron-beam drilling; TEM fabrication; hourglass profile

\section{Introduction}

The ability to manipulate materials at nanometer scales and to control the dimensions of nanostructures is a prerequisite not only of studying the novel properties of materials at different length scales but also for realizing useful miniaturized devices. The effectiveness of widely-used resist-based electron-beam lithography techniques is limited to tens of nanometers, and the top-down fabrication of sub-10 $\mathrm{nm}$ scale devices with high reproducibility is still a challenge. Transmission electron beams have long been used to study materials at nanometer scales. Upon the electron irradiation of a sample, high-energy electrons lose a portion of their kinetic energy via inelastic scattering processes in a solid, resulting in various effects including sputtering, amorphization, sublimation and desorption. Nanopores have been fabricated in thin $\mathrm{Si}_{3} \mathrm{~N}_{4}$ films using an intense electron beam from a transmission electron microscopy
(TEM) system. Nanopores embedded in an insulating membrane (e.g., $\mathrm{Si}_{3} \mathrm{~N}_{4}$ ) are a new class of nanosensors for the electrical detection and characterization of biomolecules, with single-molecule resolution [1-3]. Numerous techniques for nanopore fabrication have been developed in recent years. Based on reactive ion etching (RIE) and using a few-nm-thick textured alumina film as masking material, the transfer of a random pattern of nanopores from the masking film of alumina to a $100-n m$-thick $\mathrm{Si}_{3} \mathrm{~N}_{4}$ membrane was accomplished [4]. Electrochemical etching was used to fabricate nanopores in a silicon chip [5]. Various teams have proposed using TEM to fabricate nanopores on a $\mathrm{Si}_{3} \mathrm{~N}_{4}$ film [6-11].

\section{Experimental Method and Device Fabrication}

Apart from the performance observation and measurement, TEM can also be used as an insulating platform to form nanopores of various dimensions. This 
TEM technology is useful for the development of nano-scale applications, including nano-electronics and molecular transposition [12-15].

The proposed method is based on the irradiation of a transmission electron beam in TEM to effectively observe, etch and evaporate the membrane.

First, the top and bottom surfaces of a 4" wafer with a thickness of $500 \mu \mathrm{m}<100>$ is grown in low stress amorphous $\mathrm{Si}_{3} \mathrm{~N}_{4}$ of $100 \mathrm{~nm}$ by means of low-pressure chemical vapor deposition (LPCVD), as shown in Figure $1(a)$. Then patterning through standard photolithography and RIE is used to remove the silicon nitrade membrane to produce a square window on the back surface of the wafer. As shown in Figure 1(b) a freestanding membrane measuring $50 \times 50 \mu \mathrm{m}^{2}$ is obtained by anisotropic electrochemistry etching with $\mathrm{KCl}$ and $\mathrm{KOH}$, respectively, in contact with the front and back side of the wafer as illustrated in Figure 1(c)[2-5].

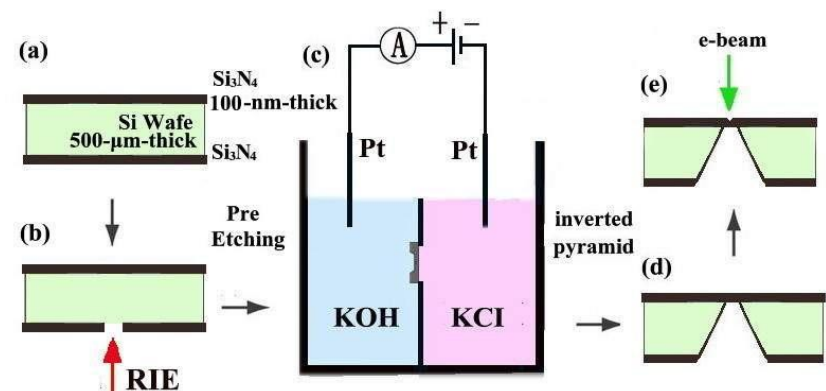

Figure 1. A schematic fabrication procedure for sample preparation:

(a) silicon nitride membrane deposited by LPCVD on both sides of the wafer

(b) a window opened on the back side with photolithography and RIE

(c) $\mathrm{KCl}$ and $\mathrm{KOH}$ were respectively in contact with the front side and back side of the wafer

(d) a freestanding membrane TEM drilling sample

(e) TEM fabrication of a nanopore

Yung-Cheng Wang received his Dr.-Ing. from the Institute of Process Measurement and Sensor Technology at the IImenau University of Technology (TU IImenau) in 2003. He is now an associate professor in the Department of Mechanical Engineering at the National Yunlin University of Science and Technology (Yuntech). His research interests include optical metrology and its applications in precision mechanical engineering, and the development of Fabry-Perot interferometers and inspection technologies for machine tools and on-line automated measurements. He has published over 50 research papers and holds 30 patents.

Dau-Chung Wang received his $\mathrm{PhD}$ in mechanical engineering from the University of California, Los Angeles, and served [JSB: "serves"?] as chairman of the Department of Mechanical Engineering and director of International Affairs in National Yunlin University of Science and Technology His main research fields include systematic design [JSB: "system design"?], control theory, micro-electromechanical systems and nanotechnology. He has published about 30 journal articles, over 60 conference papers and three textbooks.

Tsan-Chu Lin. 2014 graduated from the Institute of Engineering Science and Technology, National Yunlin University of Science [JSB: what degree?]. His dissertation focused on TEM-Based Nano-Fabrication. Since 1992 he has served as a technician at the National Changhua University of Education. He frequently lectures at the Far East Institute of Technology and has published six articles in international journal. He frequently consults on the use of penetrating mirror electron microscopy and nanometer holes.
As the silicon substrate is $\langle 100\rangle$, a crystal face etching path penetrates at an angle of $54.74^{\circ}$ to form a pyramid-shaped pore measuring $50 \times 50 \mu \mathrm{m}^{2}$, as shown in Figures 1(d, e).

\section{Hourglass Profile of a Nanopore}

Straight nanopores cannot be achieved using conventional TEM electron beam processing. Two phenomena can be observed. First, TEM can be used to depict a silicon nitride nanopore with hourglass shape [1]. Second, TEM shows the nano-silica material is drilled to create a pyramidal cross-section [2].

With TEM observation, light is concentrated around the heated area of the material. When irradiated by an electron beam at a fixed location, the temperature of the non-processing material is raised by the heat conduction to produce a graded temperature distribution. Heat concentrates on the film to produce a melting pothole, which causes the material to soften in the pit below the melting pothole.

When the temperature rises continuously and reaches its breaking point, a nanopore is generated at the bottom of a shallow crater and the material is pressed downwards to break through into hourglass-shaped nanopore as depicted. Theoretically, we could obtain a straight nanopore by reducing the size of the melted pit holes and the region of the softened materials. This could conceivably be accomplished by improving the film's thermal conductivity. A metal film is deposited on the sample to obtain the pores as depicted.

\section{Drilling Fabrication of Straight Nanopores}

\section{Fabrication Instrument}

The nanopores were fabricated using a JEOL 2010 HRTEM integrated with a Gatan digital camera for digital image processing. During the drilling process, TEM images could also provide real-time visual feedback to ensure accurate drilling control.

\section{Specimen Preparation}

Nanopores were drilled in a freestanding $\mathrm{Si}_{3} \mathrm{~N}_{4}$ membrane with a thickness of $100 \mathrm{~nm}$. The membrane was first prepared by sputtering plasma from a heat conducting gold film with a thickness of $50 \mathrm{~nm}$, as shown in Figure 2.

\section{Drilling Operation}

The JEOL 2010 HRTEM was operated at 200kV, using image magnification and the convergence angle as operational parameters to control the e-beam density. 
For image acquiring, image magnification and convergence angle were operated usually at $200 \mathrm{kX}$ and $\alpha 5$ mode, which resulted in a beam density of $60 \mathrm{pA} / \mathrm{cm}^{2}$. For nanopore drilling, it was operated at $1 \mathrm{MX}$ and $\alpha 1$ mode respectively, resulting in a more focused electron beam of $102 \mathrm{pA} / \mathrm{cm}^{2}$

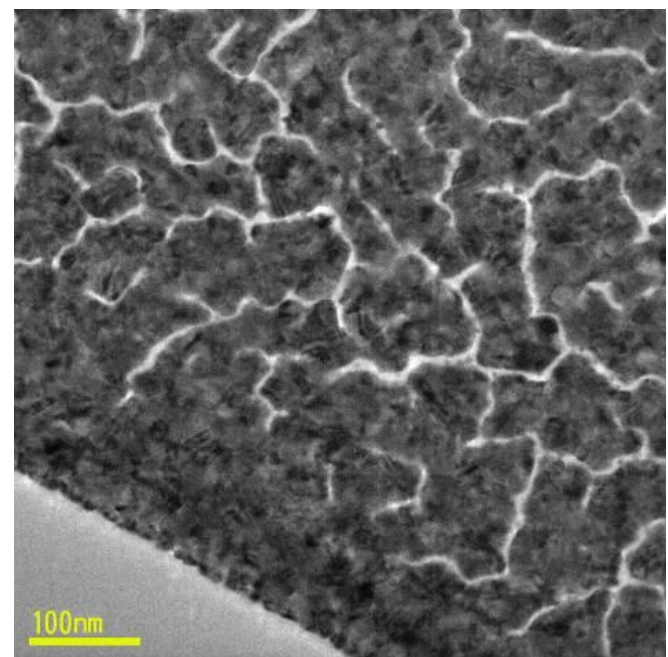

Figure 2. $\mathrm{Si}_{3} \mathrm{~N}_{4}$ membrane (bright zone) with sputtered gold film (dark zone)

\section{Results and Discussion}

An $8 \mathrm{~nm}$ nanopore (Figure $3 \mathrm{~b}$ ) was drilled in the non-gold-covered region of the $\mathrm{Si}_{3} \mathrm{~N}_{4}$ membrane (Figure 3a). The heat conduction property at the drilling spot was still enhanced by the deposition of the gold thin film nearby. Another $8 \mathrm{~nm}$ nanopore was drilled in the gold-covered region (Figures $4 a-c)$. Similarly, other nanopores measuring $3 \mathrm{~nm}, 4 \mathrm{~nm}$ and $5 \mathrm{~nm}$ were fabricated to demonstrate the practicality of the proposed technique (Figures $5 \mathrm{a}, 5 \mathrm{~b}$ and $5 \mathrm{c}$ ).

The heat conduction hypothesis could be confirmed by the transformation of the nanopore from an hourglass cross-section to a straight through pore. For the purpose of comparison, the TEM image of an hourglass nanopore was first analyzed. As outlined in Figure 6 , the hourglass nanopore profile resulted from insufficient heat conduction of the $\mathrm{Si}_{3} \mathrm{~N}_{4}$ membrane.

IMOD was used to reconstruct a three-dimensional model of the hourglass-shaped nanopore (Figure 6a) [16]. Figure $6 \mathrm{~b}$ shows the TEM top-down view of an hourglass nanopore, where the pore is shown as the brightest circle at the center of the image and is surrounded by a light grey circular area, the circumference of which indicates the rim of the upper bowl of the hourglass profile. The appearance of this circular area is seen as proof of the existence of the hourglass profile. Figure $6 \mathrm{c}$ shows the top-down view of another hourglass nanopore.
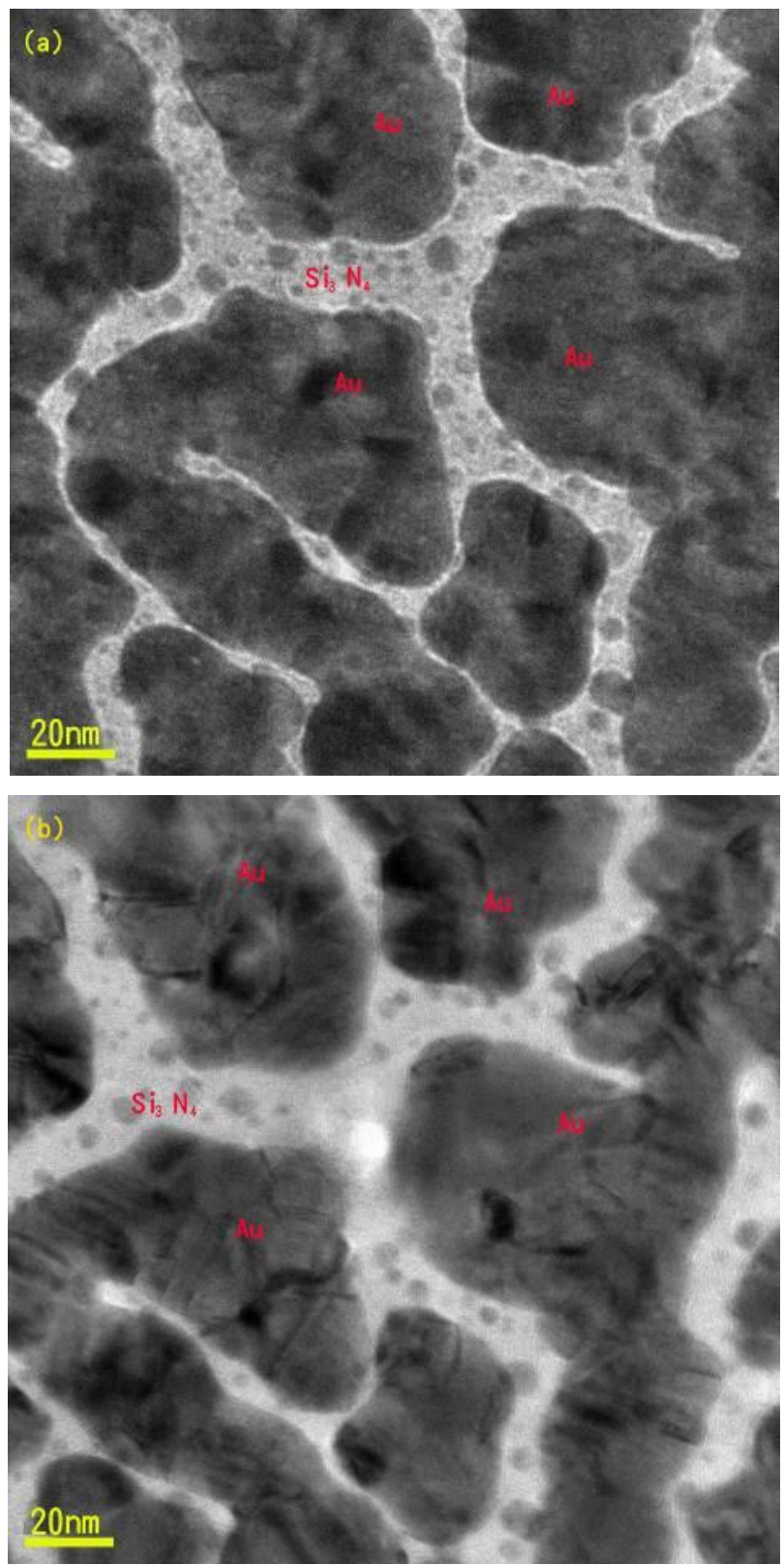

Figure 3. Nanopore drilled in non-gold-covered region: (a) before drilling, (b) after drilling.

To enhance the heat conduction, a gold thin film was deposited on the $\mathrm{Si}_{3} \mathrm{~N}_{4}$ membrane by plasma sputtering process (Figure 2). As shown in Figures 3-5, the nanopore drilled in this heat-conduction-enhanced membrane showed only as a bright circle in the TEM image, without the light grey circular area associated with the hourglass-shaped nanopore. This implies that the resulting nanopore has a straight cross-section and that such nanopores can be effectively achieved by improving the heat conduction of the $\mathrm{Si}_{3} \mathrm{~N}_{4}$ membrane. 

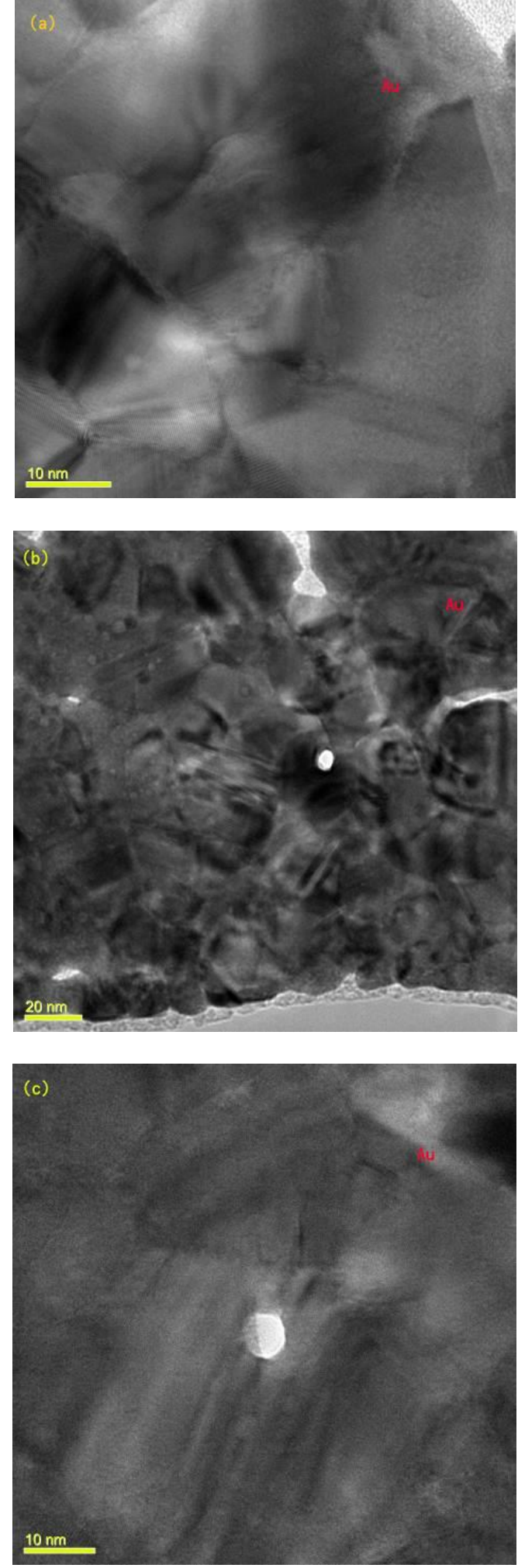

Figure 4. Nanopore drilled in the gold-covered region: (a) before drilling with a TEM magnification of $1000 \mathrm{kX}$, (b) after drilling with a TEM magnification of $400 \mathrm{kX}$, (c) after drilling with a TEM magnification of $800 \mathrm{kX}$.
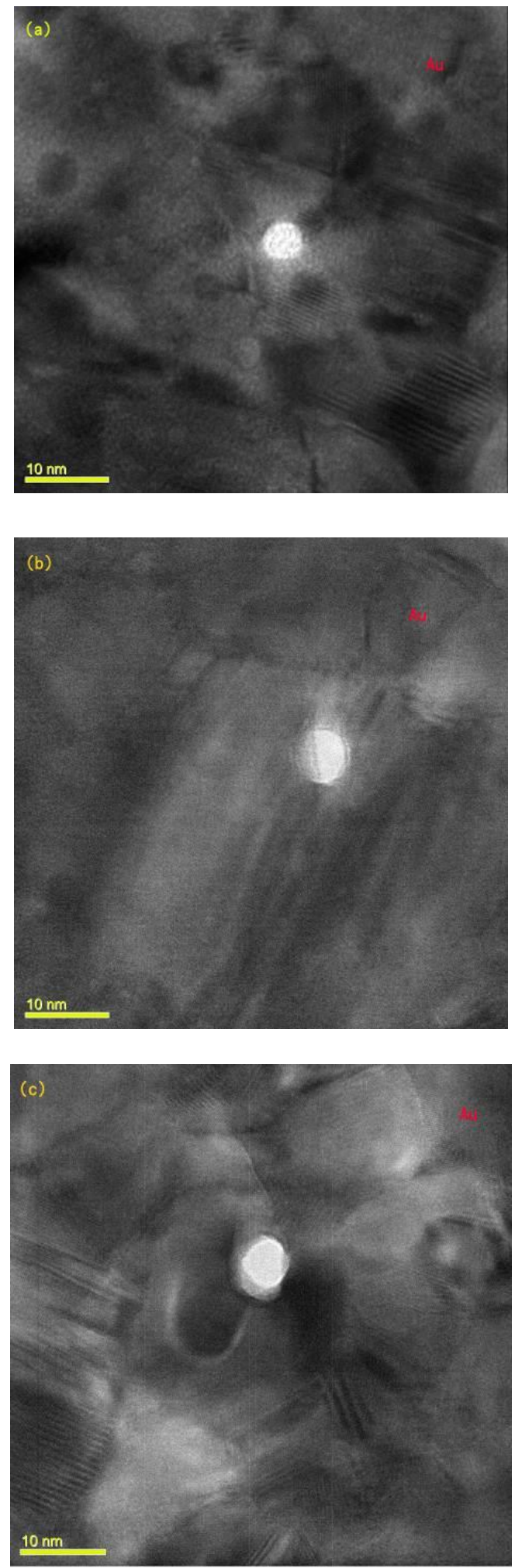

Figure 5. Nanopores of different sizes: (a) $3 \mathrm{~nm}$, (b) $4 \mathrm{~nm}$, (c) $5 \mathrm{~nm}$ 

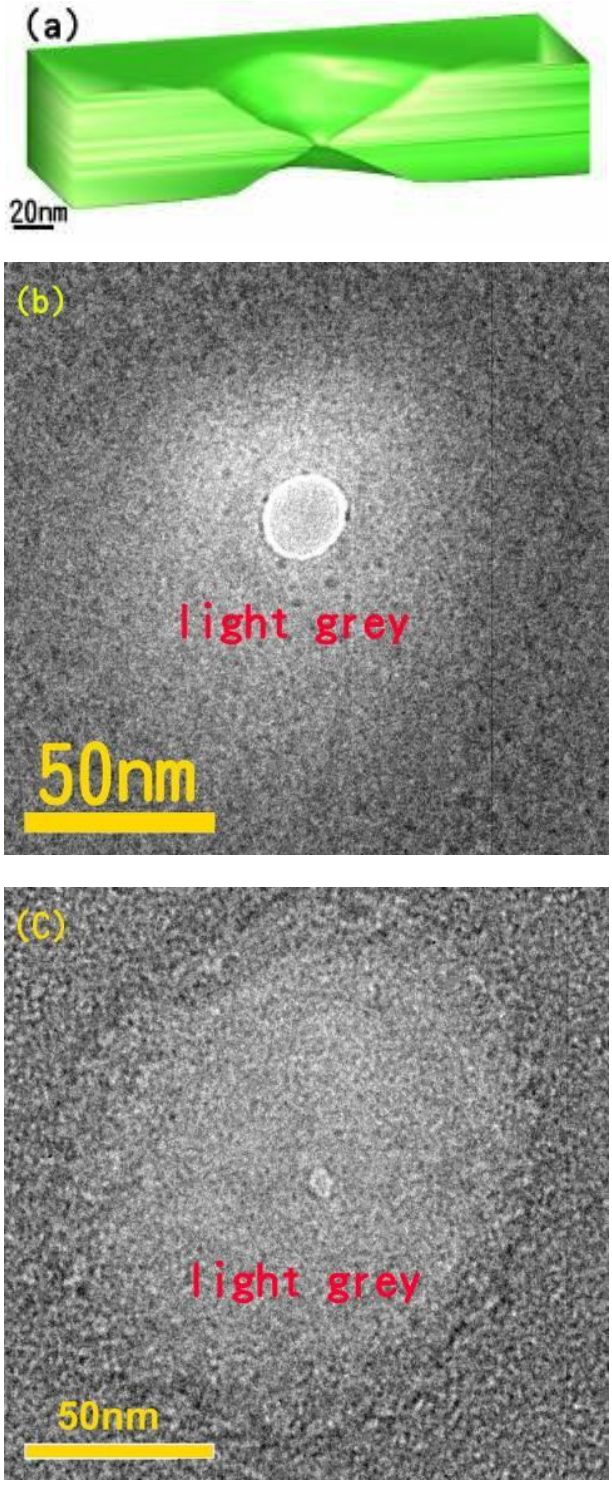

Figure 6. Hourglass nanopore: (a) three-dimensional model, (b) TEM top-down view, (c) top-down view of another hourglass nanopore

\section{Conclusions}

Improving the membrane's heat conduction was found to preserve membrane strength except for areas under direct electron collision, allowing for the drilling of straight nanopores. The deposition of gold thin film on the membrane increased the complexity of the membrane's surface tomography, thus 3D reconstructions of the straight nanopore using IMOD have yet to be accomplished, and will be the subject of future work.

\section{Acknowledgements}

This work was financially supported by Taiwan's National Science Council under grant number NSC 101-2221-E-224-027.

\section{References}

[1] M. J. Kim, B. McNally, K. Murata, and A. Meller, "Characteristics of solid-state nanometer pores fabricated using a transmission electron microscope," Nanotechnology, vol. 18, no. 20, p. 205302, 2007.

doi: $10.1088 / 0957-4484 / 18 / 20 / 205302$

[2] A. J. Storm, J. H. Chen, X. S. Ling, H. W. Zandbergen, and C. Dekker, "Fabrication of solid-state nanopores with single-nanometre precision," Nature materials, vol. 2, no. 8, pp. 537-540, 2003.

[3] A. J. Storm, C. Storm, J. Chen, H. Zandbergen, J. F. Joanny, and C. Dekker, "Fast DNA translocation through a solid-state nanopore," Nano Letters, vol. 5, no. 7, pp. 1193-1197, 2005.

doi: $10.1021 / \mathrm{nl048030d}$

[4] J. H. Chungt, X. Chen, E. J. Zimney, and R. S. Ruoff, "Fabrication of nanopores in a 100-nm thick si3n4 membrane," Journal of nanoscience and nanotechnology, vol. 6, no. 7, pp. 2175-2181, 2006.

[5] S. R. Park, H. Peng, and X. S. Ling, "Fabrication of nanopores in silicon chips using feedback chemical etching," Small, vol. 3, no. 1, pp. 116-119, 2007.

doi: $10.1002 / \mathrm{smll} .200600268$

[6] S. M. Sze, Physics of semiconductor devices. New York, USA: John Willey and Sons, 1981.

[7] T. C. Lin, R. Z. Su, Y. C. Lai, D. C. Wang, and C. S. $\mathrm{Wu}$, "Transmission electron beam drilling for nanoscale fabrication," Japanese Journal of Applied Physics, vol. 49, no. 6, p. 49, 2010. doi: $10.1143 /$ JJAP.49.06GH16

[8] S. Howorka, S. Cheley, and H. Bayley, "Sequence-specific detection of individual DNA strands using engineered nanopores," Nature Biotechnolgy, vol. 19, no. 7, pp. 636-639, 2001. doi: $10.1038 / 90236$

[9] A. Meller, L. Nivon, and D. Branton, "Voltage-driven DNA translocations through a nanopore," Physical Review Letters, vol. 86, no. 15, pp. 3435-3438, 2001.

doi: 10.1103/PhysRevLett.86.3435

[10] A. F. Sauer-Budge, J. A. Nyamwanda, D. K. Lubensky, and D. Branton, "Unzipping kinetics of double-stranded DNA in a nanopore," Physical Review Letters, vol. 90, no. 23, p. 238101, 2003. doi: 10.1103/PhysRevLett.90.238101

[11] J. Nishizawa and K. Suto, "Semiconductor raman laser," Journal of Applied Physics, vol. 51, no. 5, pp. 2429-2431, $1980 . \quad$ doi: doi:http://dx.doi.org/10.1063/1.328012 
[12] M. Y. Wu, P. Chen, U. Ziese, P. F. Alkemade, H. W. Salemink, and H. W. Zandbergen, "Tem study of locally coated nanopore fabricated by ion-beam-induced deposition in a thin membrane," Micron, vol. 41, no. 6, pp. 609-614, 2010.

doi: $10.1016 /$ j.micron.2010.03.009

[13] M. Y. Wu, D. Krapf, M. Zandbergen, H. Zandbergen, and P. E. Batson, "Formation of nanopores in a sin/sio2 membrane with an electron beam," Applied Physics Letters, vol. 87, no. 11 , pp. 113106-113106-113103, 2005. doi: 10.1063/1.2043247

[14] M. D. Fischbein and M. Drndić, "Sub-10 nm device fabrication in a transmission electron microscope," Nano Letters, vol. 7, no. 5, pp. 1329-1337, 2007. doi: $\underline{10.1021 / \mathrm{nl} 0703626}$
[15] M. J. Kim, M. Wanunu, D. C. Bell, and A. Meller, "Rapid fabrication of uniformly sized nanopores and nanopore arrays for parallel DNA analysis," Advanced Materials, vol. 18, no. 23, pp. 3149-3153, 2006.

doi: 10.1002/adma.200601191

[16] IMOD are copyright (c) 1994-2011 by the boulder laboratory for 3-dimensional electron microscopy of cells and the regents of the university of colorado. 